\title{
不对称三苯胺-锌酞菁的合成及性能研究
}

\author{
毛利军谭青龙辛冠琼韩明亮张学俊* \\ (中北大学理学院 太原 030051)
}

\begin{abstract}
摘要 以对氨基酚、对硝基氯苯、8-羊基喹啉、4-硝基邻苯二甲腈以及醋酸锌为主要原料，合成了不对称 2(3)-[二(对硝 基苯基)氨基苯氧基]-9(10),16(17),23(24)-三(8-喹啉氧)锌酞菁(TQPc). 用核磁、红外、元素分析等表征 TQPc 以及前驱化 合物结构, TQPc 的电子吸收光谱表现出了强烈的 $\pi$ - $\pi$ *跃迁现象. 利用紫外光谱研究了其 $N, N-$ 二甲基甲酰胺(DMF)和 $\mathrm{CH}_{2} \mathrm{Cl}_{2}$ 溶液的吸收光谱性质, 结果表明在 $\mathrm{DMF}$ 中主要以单体的形式存在, 浓度在 $0.223 \times 10^{-5} \sim 2.587 \times 10^{-5} \mathrm{~mol} / \mathrm{L}$ 时, $\mathrm{TQPc}$ 在 $\mathrm{CH}_{2} \mathrm{Cl}_{2}$ 溶液中有二聚体的存在, 经计算得到平衡常数为 $0.24 \times 10^{5} \mathrm{~L} / \mathrm{mol}$. 用循环伏安法研究了 $\mathrm{TQPc}$ 的氧化还 原行为, 结合差分伏安数据计算了能级结构, LUMO (-1.04 Vvs SCE) 和 HOMO (0.78 Vvs SCE) 与纳米 $\mathrm{TiO}_{2}$ 导带能 级匹配，可作为性能较好的电荷传输材料用于染料敏化太阳能电池.
\end{abstract}

关键词 酞菁; 8-羊基喹啉; 三苯胺; 光敏染料; 二聚体

\section{Synthesis and Study of Properties for Asymmetric Triphenylamine-Zinc Phthalocyanine}

\author{
Mao, Lijun Tan, Qinglong Xin, Guanqiong Han, Mingliang Zhang, Xuejun* \\ (Department of Chemistry, College of Science, North University of China, Taiyuan 030051)
}

\begin{abstract}
Triphenylamine-zinc phthalocyanine (TQPc) that contains a bulky triphenylamine group and three 8-oxy-quinoline groups has been found to exhibit preferable performance. The first example of TQPc has been synthesized. Its zinc complexes of the well-known electron transport material displays enhanced electron-accepting ability relative to free- metallophthalocyanines, and can also be used as chemical sensors, liquid crystals, photodynamic therapy, data storage and non-linear optics. The triphenylamine-zinc phthalocyanine is synthesized by statistical condensation. The main chemicals are aminophenol, p-chloronitrobenzene, 8-hydroxyquinoline, 4-nitrophthalonitrile and zinc acetate. The fragment 4,4'-dinitro-4"-hydroxytriphenylamine is synthesized by the reacting from aminophenol with $p$-chloronitrobenzene at the catalysis of anhydrous potassium carbonate. The gross yield is $27.5 \%$, and its purity is $96.6 \%$. In comparison with other methods, there are some advantages in this method: the materials are cheap and easily available, and the product can be used as a substituent for phthalocyanine. All compounds are characterized by ${ }^{1} \mathrm{H}$ NMR, IR spectra and elemental analysis. The electronic spectra of TQPc exhibits an intense $\pi-\pi^{*}$ transition of triphenylamine unit identity together with characteristic B bands of the phthalocyanine core. Energy transfer through oxy bridges has been confirmed by ultraviolet irradiation of triphenylamine. The aggregative behavior is studied in DMF and $\mathrm{CH}_{2} \mathrm{Cl}_{2}$. The results indicate that it is not aggregative in DMF, whereas forms dimer in $\mathrm{CH}_{2} \mathrm{Cl}_{2}$ at $0.223 \times$ $10^{-5} \sim 2.587 \times 10^{-5} \mathrm{~mol} / \mathrm{L}$. The equilibrium constant for the dimer is calculated at the same time, indicating that the less polar solvent is unfavorable for the presence of the monomer. The redox behavior is studied by cyclic voltammetry. Its level structure of energy band is calculated by cyclic voltammetry combined with differential voltammograms, this molecule has been found to have a low LUMO ( $-1.04 \mathrm{~V} v s \mathrm{SCE})$ and a deep HOMO $(0.78 \mathrm{~V} v s$ SCE) energy level. The results indicate that it matches with the energy level of the nanocrystalline of $\mathrm{TiO}_{2}$, and is thus very promising as an electron transport material for dye-sensitized solar cells.
\end{abstract}

Keywords phthalocyanine; 8-hydroxyquinoline; triphenylamine; photosensitive dyes; dimer

染料敏化太阳能电池 (dye-sensitized solar cells,

DSSCs)主要由纳米多孔薄膜、光敏染料、电解质和对电

\footnotetext{
*E-mail: zhangxuejun@nuc.edu.cn

Received June 19, 2012; revised August 1, 2012; published online August 10, 2012.

Project supported by the National Natural Science Foundation of China (No. 20871108) and the Natural Science Foundation of Shanxi Province (No. 2011011022-4).

国家自然科学基金(No. 20871108)、山西省自然科学基金(No. 2011011022-4)资助项目.
} 
极组成 ${ }^{[1 \sim 3]}$. 用于 DSSCs 的染料主要有金属有机和纯有 机染料两大类, 纯有机染料成本低, 吸光系数高, 便于 进行分子结构设计, 如二氢吲哚 ${ }^{[4]}$ 、蒽醌 ${ }^{[5]}$ 、三苯胺类 ${ }^{[6]}$ 、 酞菁、卟啉 ${ }^{[7,8]}$ 等, 但其长期稳定性较低, 且组装的 DSSCs 的光电转换效率有待提高. 金属有机染料以联 吡啶钓为代表, 具有非常高的化学稳定性和良好的可见 光谱响应特性, 组装的 DSSCs 保持着目前最高的光电 转换效率, 但是钉在自然界含量及其稀少, 价格昂贵 ${ }^{[9]}$. 在纯有机染料领域里卟啉 ${ }^{[10 ~ 13]}$ 和唒菁受到了较大的关 注，其中金属酞菁是一类性能优异且应用广泛的功能材 料, 由于金属酞菁有 18 个共轭 $\pi$ 电子, 使其具有许多独 特的物理化学性质, 近年来引起人们越来越浓厚的研究 兴趣.

对金属酞菁的合成和应用研究大多以对称结构的 酞菁为主, 而有关不对称金属酞菁的报道和研究很少. 不对称的金属酞菁由于结构上的特殊性, 表现出许多独 特的性能, 如当在酞菁的苯环上同时引入供电子基团和 吸电子基团后, 其三阶非线性光学效应更为优越, 产生 二阶非线性光学效应等 ${ }^{[14]}$; 以酞菁为功能性基团与其 他功能材料如三苯胺相连接, 也涉及到先合成一些本身
带反应性官能团(比如硝基、羟基、氨基等)的不对称酞 菁, 而后再以酞菁为构筑单元与其他的功能性分子相连 接 ${ }^{[15]}$. 当两种邻苯二腈 $(\mathbf{A}$ 和 $\mathbf{B})$ 缩合成酞菁时会得到 6 种酞菁配合物 $\left(\mathbf{A}_{4}, \mathbf{A}_{\mathbf{3}} \mathbf{B}_{1}, \mathbf{A A B B}, \mathbf{A B A B}, \mathbf{A B}_{3}, \mathbf{B}_{4}\right)$; 如 $\mathbf{A}$ 和 $\mathbf{B}$ 的物质的量之比为 $3: 1$ 时, 理论上缩合得到的产 物统计为 $\mathbf{A}_{\mathbf{4}}$ (33\%), $\mathbf{A}_{3} \mathbf{B}(44 \%)$, 其他产物各自的含量较 少, 本文采用统计缩合法设计合成了含三苯胺、喹啉氧 基团的不对称锌酞菁.

Campbell 等 ${ }^{[16]}$ 研究了不同取代基的锌酞菁和铝酞 菁在 DSSCs 中的应用，但针对含有两种发色基团如三 苯胺、喹啉的不对称二元染料的取代基对 Q 带和 B 带 最大吸收峰位置的影响, 以及相应的氧化还原电势、聚 集性等方面的研究未见报道. 本实验以对氨基酚、对硝 基氯苯、8-差基喹啉、4-硝基邻苯二甲腈以及醋酸锌为 主要原料, 采用统计缩合法合成了中心离子为锌的金属 酞菁(TQPc) (Scheme 1), 结构新颖, 性能优良. 研究了 其光物理与电化学性质, 利用循环伏安法研究了氧化还 原行为, 并结合差分伏安法计算了其能级结构, 表明 TQPc 是一种性能较好的光敏染料.<smiles>COc1cccc2cccnc12</smiles><smiles>COC(=O)c1ccc([N+](=O)[O-])cc1CC(C)(C)O</smiles><smiles>CCCCCOc1cccc2cccnc12</smiles>

Scheme 1 


\section{1 结果与讨论}

\section{1 合成}

四取代酞菁一般是由多种同分异构体组成的混合 物, 研究表明其同分异构体在理论上存在一定的比例, 受中心金属离子、反应条件等因素的影响会发生变 化 [17 19]. 本文用柱层析法分离提纯产物, 得到的是 TQPc 的多种同分异构体. 首先合成三苯胺类化合物二(对硝 基苯基)氨基苯酚(1), 再与 4-硝基邻苯二甲腈反应得到 前驱分子碎片(2), 从而将三苯胺基团接到酞菁大环上. 研究表明三苯胺基团及其衍生物具有很强的给电子能 力, 而且其中的 3 个苯环具有非共平面结构, 可以防止 分子之间的 $\pi-\pi$ 堆积, 减少染料的聚集 ${ }^{[20]}$, 因此, 将二 (对硝基苯基)氨基苯酚(1)作为取代基接到酞菁大环上 不但可以增加其电子云密度而且可减少聚集性, 促进其 在一般溶剂中的溶解性. 三苯胺类化合物也是一种性能 较好的光敏染料, 具有较高的空穴迁移率和良好的传输 性能, 广泛应用于光电材料和空穴传输材料, 在紫外区 对太阳光谱有较强的吸收 ${ }^{[20]}$, 可与酞菁的 B 吸收带相叠 加而使其吸收强度增大. 研究表明不对称酞菁化合物有 许多独特的性质, 如较好的溶解性、光物理性能和电化 学性质等, 基于此, 实验同时在酞菁环上引入喹啉基团, 得到不对称结构的二元光敏染料 TQPc, 经测试具有较 好的光谱吸收等特性, 为进一步设计性能更好的酞菁染 料的分子结构提供了思路, 也为制备光电转化率更高的 染料敏化太阳能电池奠定了基础.

\section{$1.2 \mathrm{TQPC}$ 的溶液性质}

由于 TPQc 外围苯环上有 3 个 8 -喹啉氧基和 1 个三 苯胺基, 有效地提高了其在有机溶剂中的溶解性, 在 DMF、二氯甲烷、四氢呋喃和二甲基亚砜(DMSO)等常 见溶剂中都有良好的溶解性, 这可能和取代基在这些有 机溶剂中有较好的溶解性有关. 此外, 空间位阻效应降 低了其 $\pi-\pi$ 相互作用 ${ }^{[18]}$, 阻碍了二聚体甚至多聚体的形 成, 因而在一些有机溶剂中不但表现出更好的溶解性, 而且在一定浓度范围内主要以单体的形式存在. 本文主 要研究了其 $\mathrm{DMF}$ 和 $\mathrm{CH}_{2} \mathrm{Cl}_{2}$ 溶液的吸收光谱性质.

多数金属酞菁在溶液中容易发生聚集, 研究表明聚 集平衡常数 $K$ 、聚集度 $n$ 以及摩尔吸光系数 $\varepsilon$ 的关系式 可表示为 ${ }^{[14]}$ :

$$
\varepsilon_{\mathrm{e}}=\varepsilon^{-} \frac{n K}{\varepsilon^{n-1}} \varepsilon_{\mathrm{e}}^{n} \cdot c_{0}^{n-1}
$$

$\varepsilon_{\mathrm{e}}$ 为有效摩尔吸光系数, $c_{0}$ 为酞菁单体初始时的浓度.

在一定浓度范围内以 $\varepsilon_{\mathrm{e}}$ 对 $\varepsilon_{\mathrm{e}}^{n} \cdot c_{0}^{n-1}$ 作图, 取不同的 $n$ $(n=2,3,4$ 等)值代入 Eq. 1 , 当函数轨迹为直线时所选取 的 $n$ 值就为聚集度.
分别配制了 8 种不同浓度的 DMF, $\mathrm{CH}_{2} \mathrm{Cl}_{2}$ 溶液, 浓 度范围为 $0.1 \times 10^{-5} \sim 2.6 \times 10^{-5} \mathrm{~mol} / \mathrm{L}$, 测定了各自的吸 收光谱, 见图 1 和图 2(图中箭头方向表示溶液浓度逐渐 减小), 研究了 $\mathrm{TQPc}$ 在每种溶液中的聚集状态, 具体数 据见表 1 和表 2.

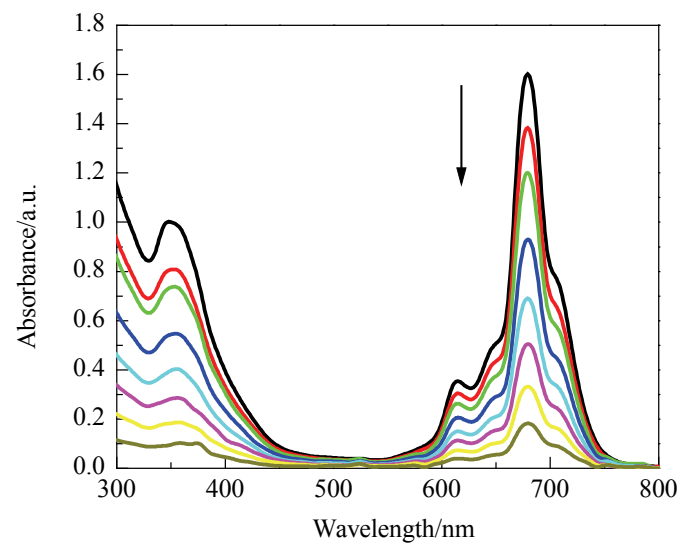

图 $1 \mathrm{TQPc}$ 在 DMF 中不同浓度的吸收光谱

Figure 1 Absorption spectra of TQPc in DMF

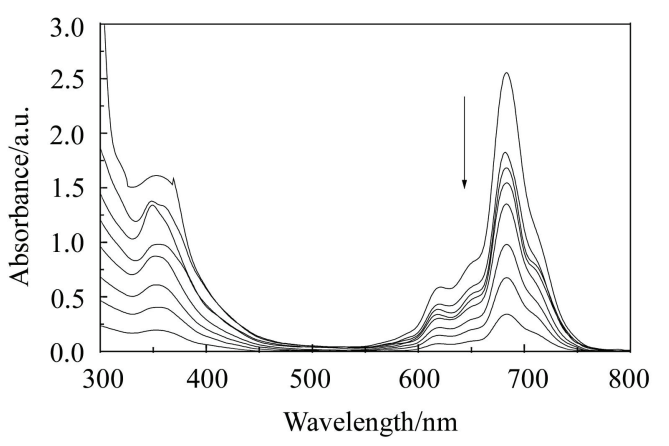

图 $2 \mathrm{TQPc}$ 在 $\mathrm{CH}_{2} \mathrm{Cl}_{2}$ 中不同浓度的吸收光谱

Figure 2 Absorption spectra of TQPc in $\mathrm{CH}_{2} \mathrm{Cl}_{2}$

表 $1 \mathrm{TQPc}$ 在 DMF 中不同浓度的光谱性质

Table 1 Spectral property of TQPc in DMF $\left(\lambda_{\max }=679 \mathrm{~nm}\right)$

\begin{tabular}{cccc}
\hline Entry & $c_{0} /\left(\mathrm{mol} \cdot \mathrm{L}^{-1}\right)$ & $A$ & $\varepsilon_{\mathrm{e}} /\left(\mathrm{L} \cdot \mathrm{mol}^{-1} \cdot \mathrm{cm}^{-1}\right)$ \\
\hline 1 & $1.160 \times 10^{-5}$ & 1.578 & $1.36 \times 10^{5}$ \\
2 & $1.014 \times 10^{-5}$ & 1.359 & $1.34 \times 10^{5}$ \\
3 & $0.861 \times 10^{-5}$ & 1.180 & $1.37 \times 10^{5}$ \\
4 & $0.706 \times 10^{-5}$ & 0.960 & $1.36 \times 10^{5}$ \\
5 & $0.492 \times 10^{-5}$ & 0.669 & $1.36 \times 10^{5}$ \\
6 & $0.370 \times 10^{-5}$ & 0.499 & $1.35 \times 10^{5}$ \\
7 & $0.232 \times 10^{-5}$ & 0.316 & $1.36 \times 10^{5}$ \\
8 & $0.132 \times 10^{-5}$ & 0.178 & $1.35 \times 10^{5}$ \\
\hline
\end{tabular}

由表 1 可知 TQPc 在 DMF 中的有效摩尔吸光系数 $\varepsilon_{\mathrm{e}}$ 不随浓度 $c_{0}$ 的变化而变化, 基本为一恒定值, 说明在此 浓度范围内 TQPc 在 DMF 中不发生聚集现象, 完全以单 体的形式存在，但从表 2 的数据可以看出, 在 $\mathrm{CH}_{2} \mathrm{Cl}_{2}$ 中 有效摩尔吸光系数 $\varepsilon_{\mathrm{e}}$ 却随浓度的变化而变化, 说明其中 
表 $2 \mathrm{TQPc}$ 在 $\mathrm{CH}_{2} \mathrm{Cl}_{2}$ 中不同浓度的光谱性质

Table 2 Spectral property of TQPc in $\mathrm{CH}_{2} \mathrm{Cl}_{2}\left(\lambda_{\max }=683 \mathrm{~nm}\right)$

\begin{tabular}{|c|c|c|c|c|}
\hline Entry & $c_{0} /\left(\mathrm{mol} \cdot \mathrm{L}^{-1}\right)$ & $A$ & $\begin{array}{c}\varepsilon_{\mathrm{e}} / \\
\left(\mathrm{L} \cdot \mathrm{mol}^{-1} \cdot \mathrm{cm}^{-1}\right)\end{array}$ & $\begin{array}{c}\varepsilon_{\mathrm{e}}^{2} c_{0} / \\
\left(\mathrm{L} \cdot \mathrm{mol}^{-1} \cdot \mathrm{cm}^{-2}\right)\end{array}$ \\
\hline 1 & $2.587 \times 10^{-5}$ & 2.509 & $0.970 \times 10^{5}$ & $2.434 \times 10^{5}$ \\
\hline 2 & $1.644 \times 10^{-5}$ & 1.784 & $1.085 \times 10^{5}$ & $1.935 \times 10^{5}$ \\
\hline 3 & $1.470 \times 10^{-5}$ & 1.634 & $1.112 \times 10^{5}$ & $1.818 \times 10^{5}$ \\
\hline 4 & $1.331 \times 10^{-5}$ & 1.511 & $1.135 \times 10^{5}$ & $1.715 \times 10^{5}$ \\
\hline 5 & $1.110 \times 10^{-5}$ & 1.307 & $1.177 \times 10^{5}$ & $1.538 \times 10^{5}$ \\
\hline 6 & $0.743 \times 10^{-5}$ & 0.936 & $1.260 \times 10^{5}$ & $1.180 \times 10^{5}$ \\
\hline 7 & $0.470 \times 10^{-5}$ & 0.630 & $1.342 \times 10^{5}$ & $0.846 \times 10^{5}$ \\
\hline 8 & $0.223 \times 10^{-5}$ & 0.319 & $1.430 \times 10^{5}$ & $0.456 \times 10^{5}$ \\
\hline
\end{tabular}

存在聚集平衡, 根据 Eq. 1 作它们的状态图, 发现当 $n=$ 2 时函数为线性关系(图 3), 由此可见 TQPc 在 $\mathrm{CH}_{2} \mathrm{Cl}_{2}$ 中的聚集体为二聚体. 从图 3 的直线截距和斜率可求出 单体的摩尔吸光系数 $\varepsilon=1.48 \times 10^{5} \mathrm{~L} \cdot \mathrm{mol}^{-1} \cdot \mathrm{cm}^{-1}$ 和二聚

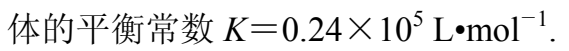

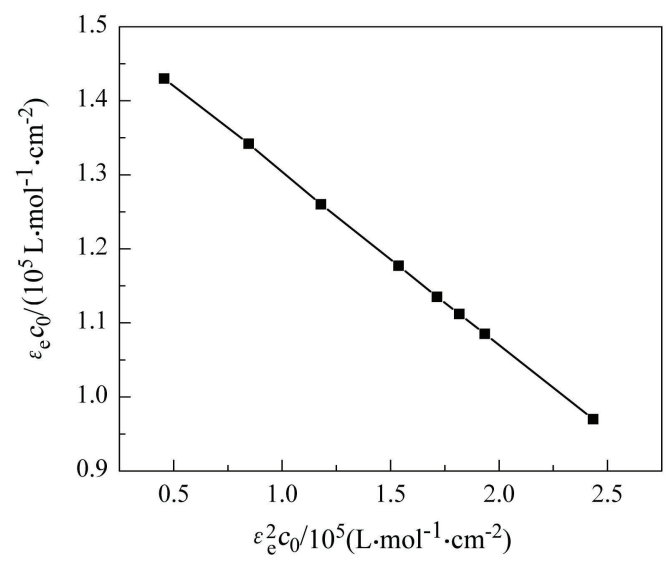

图 3 在 $\mathrm{CH}_{2} \mathrm{Cl}_{2}$ 中不同浓度金属酞菁的聚合平衡图

Figure 3 Aggregitive equilibrium digram in $\mathrm{CH}_{2} \mathrm{Cl}_{2}$

通过以上实验发现 TQPc 在极性较大的溶剂 DMF 中以单体形式存在, 而在极性较小的溶剂 $\mathrm{CH}_{2} \mathrm{Cl}_{2}$ 中有 二聚体存在, 说明溶剂的极性大有利于其单体的存在. 从 TQPc 的 DMF 和 $\mathrm{CH}_{2} \mathrm{Cl}_{2}$ 溶液的 UV-Vis 光谱图可知, 它们的特征 $Q$ 带分别位于 679 和 $683 \mathrm{~nm}$, 相对与无取代 金属酞菁锌 $670 \mathrm{~nm}$ 明显红移 ${ }^{[21]}$, 表明 TQPc 将会是一种 很有潜力的光敏剂. B 带都位于 $355 \mathrm{~nm}$ 左右. $\mathrm{CH}_{2} \mathrm{Cl}_{2}$ 溶 液的吸收谱带相对 DMF 有 $4 \mathrm{~nm}$ 的红移, 可能是 $\mathrm{CH}_{2} \mathrm{Cl}_{2}$ 的介电常数和极性都小于 $\mathrm{DMF}^{[22]}$ 而导致 $\mathrm{TQPc}$ 的吸收 谱带发生红移现象; 染料的聚集性是由分子间的强偶合 作用造成的, 会引起吸收光谱的红移或蓝移现象, 研究

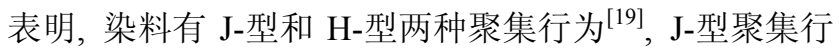
为会引起红移现象, 蓝移现象一般是由 $\mathrm{H}$-型聚集现象 引起的, 由此说明 TQPc 在 $\mathrm{CH}_{2} \mathrm{Cl}_{2}$ 中产生了 $\mathrm{J}$-型聚集现 象. 由 TQPc 的吸收光谱图可知, 其在 $355 \mathrm{~nm}$ 左右有强
而明显的吸收峰，这是由三苯胺基团与酞菁的 B 吸收带 相叠加而造成的. 从 UV-Vis 光谱图可知, 三苯胺和喹 啉取代基与平面结构的酞菁大环通过氧桥相结合, 由于 $\pi-\pi, p-\pi$ 共轭效应，它们的 $\pi$ 电子可以进入到酞菁大环 形成了更大的可流动的 $\pi$ 电子体系 ${ }^{[23]}$, 从而使 $\pi-\pi$ *能级 差减小, 产生红移现象 ${ }^{[19,23,24]}$, 并且 TQPc 结构上不对称 使电子云分布不均从而表现出优越的光学特性, 使其更 能被很好的用于 DSSCs.

\subsection{TQPc 的电化学性质}

图 4 为 TQPc 在 $\mathrm{DMF}$ 中的循环伏安曲线, 溶液浓度 为 $1 \times 10^{-5} \mathrm{~mol} / \mathrm{L}$, 所用电解质为高氯酸四丁基铵 (TBAP, $0.1 \mathrm{~mol} / \mathrm{L})$, 采用三电极体系, 其中工作电极和 对电极为铂电极, 甘采电极为参比电极, 扫描速度 100 $\mathrm{mA} / \mathrm{s}$, 扫描范围为 $-2 \sim 2 \mathrm{~V}$. 从循环伏安图中可测得阴 极峰电流 $i_{\mathrm{p}, \mathrm{c}}$ 和峰电位 $\varphi_{\mathrm{p}, \mathrm{c}}$ 以及阳极峰电流 $i_{\mathrm{p}, \mathrm{a}}$ 和峰电位 $\varphi_{\mathrm{p}, \mathrm{a}}$. 对于绝对的可逆反应，曲线上下对称，此时上下峰 电流的比值和峰电位的差值分别为 ${ }^{[25]}$.

$$
\begin{aligned}
& \left|i_{\mathrm{p}, \mathrm{a}} / i_{\mathrm{p}, \mathrm{c}}\right| \approx 1 \\
& \Delta \varphi=\varphi_{\mathrm{p}, \mathrm{a}}-\varphi_{\mathrm{p}, \mathrm{c}}=\frac{2.2 R T}{z F}=\frac{56}{z} m V\left(25{ }^{\circ} \mathrm{C}\right)
\end{aligned}
$$

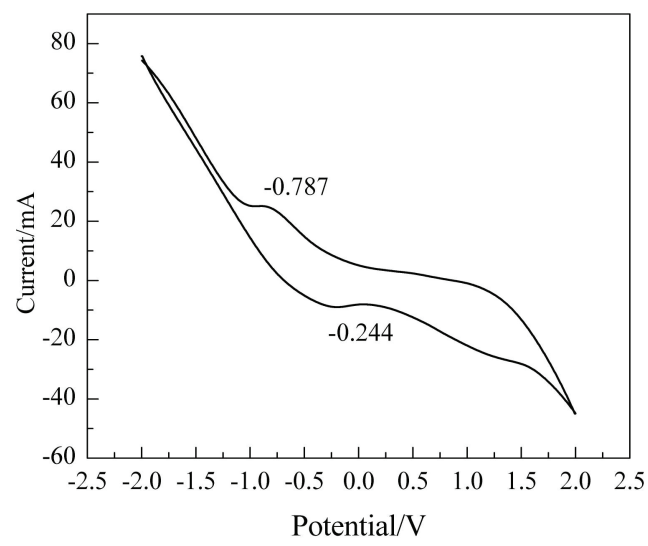

图 $4 \mathrm{TQPc}$ 在 DMF 中的循环伏安曲线

Figure 4 Cyclic voltammogram of TQPc in DMF

从图 4 可以看出, 在 -0.787 和 $-0.244 \mathrm{~V}$ 处有一对 明显的氧化还原峰, 半波电位为 $E_{1 / 2}=-0.516 \mathrm{~V}$, 此时 $\left|i_{\mathrm{p}, \mathrm{a}} / i_{\mathrm{p}, \mathrm{c}}\right|=0.85$, 表明其氧化还原反应是一个准可逆过 程, 有许多学者都曾经深入研究过金属酞菁的氧化还原 特性, 研究表明, 镍、铜、锌酞菁的氧化还原过程仅仅 发生在唒菁大环上 ${ }^{[26]}$, 因此, TQPc 的氧化还原峰源于酞 菁大环. 由此可以看出所合成的锌酞菁具有特定的电化 学活性. 这为进一步研究其光电性质提供了基础. 


\section{$1.4 \mathrm{TQPC}$ 的能级结构的研究}

酞菁作为一种有机半导体材料, 其能级结构可用吸 收光谱法表征 ${ }^{[17,27]}$. 带隙 $E_{0-0}$ 通常是由 UV-Vis 光谱的低 能带吸收边来确定, 也可采用吸收光谱低能带吸收峰值 波长来计算. 但是, 采用紫外吸收光谱法只能得到带隙 值而无法确定其 HOMO 和 LUMO 的绝对值. 电化学方 法可用于表征有机半导体材料的能带结构, 在同时进行 氧化和还原反应的前提下可以计算出有机半导体材料 的全部能带结构参数.

根据据酞菁 DMF 溶液的 UV-Vis 光谱可得到酞菁 的能带隙 $E_{0-0}$, 由 Eq. (4) 所得 TQPc 的 $E_{0-0}$ 为 $1.82 \mathrm{eV}$, 能 带隙比无取代锌酞菁 ${ }^{[21]}$ 降低 $0.03 \mathrm{eV}$, 比之前所合成的 $\mathrm{PPC}$ 和 $\mathrm{QPC}^{[27]}$ 也降低了 $0.01 \mathrm{eV}$, 表明 $\mathrm{TQPc}$ 体系电子相 比无取代锌酞菁以及 PPC 和 QPC 更易进行 $\pi-\pi *{ }^{*}$ 跃迁 ${ }^{[24]}$, 从而表现出了更好的性能.

$$
E_{0-0}=\frac{1240}{\lambda_{\max }}
$$

式中 $\lambda_{\text {max }}$ 为该酞菁的最大吸收波长.

酞菁的基态氧化电位 $\left(E_{1 / 2(\mathrm{ox})}\right)$ 可以通过循环伏安法 的半波电位 $\left[\left(E_{1 / 2}\right)\left(E_{\mathrm{ox}}-E_{\mathrm{red}}\right) / 2\right]$ 或者差分脉冲伏安法的 顶点电位得到 ${ }^{[28 ~ 31]}, E_{1 / 2(\mathrm{ox})}$ 对应的是酞菁的 HOMO 能 级, 因此从图 5 中可以读出其 HOMO 能级, 由酞菁的 $E_{1 / 2(\mathrm{ox})}$ 和 $E_{0-0}$, 结合 Eq. (5) ${ }^{[27]}$ 可计算出激发态氧化电位 $E^{*}$ (对应 LUMO 能级).

$$
E^{*}=E_{1 / 2(\mathrm{ox})}-E_{0-0}
$$

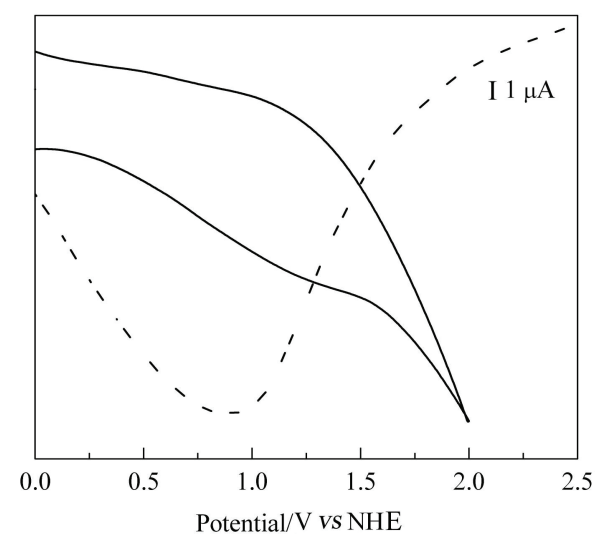

图 $5 \mathrm{TQPc}$ 循环伏安图(一)和差分脉冲(---)

Figure 5 Cyclic (-) and differential pulse voltammograms (---) of TQPc.

由图 5 可知 $\mathrm{TQPc}$ 在 $0.78 \mathrm{~V}$ (HOMO 能级)处呈现出 明显的准可逆氧化峰, 结合其能带隙便可计算出其激发 态 (LUMO 能级)的能级为 $-1.04 \mathrm{~V}$. 而纳米 $\mathrm{TiO}_{2}$ 导带的 电势为 $-0.74 \mathrm{~V} v s \mathrm{SCE}^{[27]}$, 由此可知 TQPc 的 $\mathrm{LUMO}$ 电 势低于 $\mathrm{TiO}_{2}$ 导带, 可保证电子从激发态到 $\mathrm{TiO}_{2}$ 导带的
有效注入 ${ }^{[18]}$. 此外, TQPc 的 HOMO 电势高于氧化还原 电对 $\mathrm{I}^{-} / \mathrm{I}_{3}^{-}(0.2 \mathrm{~V} v s \mathrm{SCE})$, 这样可以满足失电子的 $\mathrm{TQPc}^{+}$从 $\mathrm{I}^{-}$得到电子而再生. 图 10 是 $\mathrm{TQPc}$ 的能级分布 图. 从图 6 中可以看出其与 $\mathrm{TiO}_{2}$ 导带能级匹配, 可作为 染料敏化剂用于 DSSCs 中.

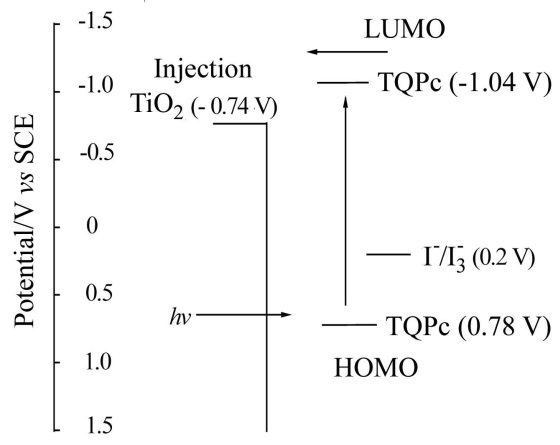

图 6 能级分布图

Figure 6 Energy level diagram for TQPc

\section{2 结论}

采用概率统计的方法合成了三苯胺、喹啉基团取代 的 $1: 3$ 型不对称锌酞菁 $\mathrm{TQPc}$, 这种新型不对称二元光 敏染料以酞菁为主体, 在酞菁环上引入了其他的发色基 团，使取代基的 $\pi$ 电子也可进入酞菁大环而形成一个完 整的体系, 在一定程度上改变了其光物理与电化学性 质，比无取代锌酞菁以及之前合成的不对称锌酞菁 PPC 和 $\mathrm{QPC}$ 的带隙更低, 使电子进行 $\pi-\pi$ * 跃迁所需的能量 进一步减少而产生红移现象. 由于较大取代基三苯胺、 喹啉基团所产生的空间位阻而表现出较好的溶解性，使 $\mathrm{TQPc}$ 溶于一般酞菁较难溶解的 $\mathrm{CH}_{2} \mathrm{Cl}_{2}$. 其能级结构与 $\mathrm{TiO}_{2}$ 导带能级匹配, 有望用于开发性能优良的染料敏化 太阳能电池.

\section{3 实验部分}

\section{1 原料}

所用主要原料, 对氨基酚、对硝基氯苯、8-羟基喹 啉均从阿拉丁试剂网订购，纯度都为分析纯; 4-硝基邻 苯二甲腈购买于石家庄埃法化学科技有限公司; 醋酸锌 (天津市光复精细化工研究所), 高氯酸四丁基铵, $N, N$ 二甲基甲酰胺 $(N, N$-dimethylformamide, DMF, 国药集团 化学试剂有限公司)也均为分析纯, 使用前未进一步提 纯.

\section{2 主要分析仪器}

核磁共振仪: Unity-400, 美国 Varian Inova 公司; 元素分析仪: Perkin-Elmer 2400, 美国 PE 公司; 紫外-可 见分光光度计: UV-2300, 上海天美仪器有限公司; CHI 
电化学分析仪: LK2005A, 天津市兰力科化学电子高技 术有限公司; 红外光谱仪: FI-TR4800S, 日本岛津研究 所; 真空干燥箱: DZF-6020, 河南巩义市予华仪器有限 责任公司; 旋转蒸发器: RE-52C, 河南巩义市予华仪器 有限责任公司; 数字显示显微熔点测定仪: X-4, 北京泰 克仪器有限公司。

\section{3 实验步骤}

\subsection{1 “前驱分子碎片” 的合成}

二(对硝基苯基)氨基苯酚(1)的合成与表征: 在三口 烧瓶中加入对硝基氯苯 (3 g, $0.019 \mathrm{~mol}$ ), 对氨基苯酚(1 $\mathrm{g}, 0.0095 \mathrm{~g})$, 碳酸钾 $(3.5 \mathrm{~g})$. 用量筒量取 $12 \mathrm{~mL}$ 二甲基 亚砜, $8 \mathrm{~mL}$ 二甲苯, 加入到三口烧瓶中, 安好冷凝管, 搅拌回流 $14 \mathrm{~h}$, 反应温度 $176{ }^{\circ} \mathrm{C}$, 得到颗粒物后反复水 洗、酸洗、抽滤、干燥、重结晶, 得棕黄色化合物 1 . 产 量 $1.1 \mathrm{~g}$, 产率 $27.5 \%$. m.p. $216{ }^{\circ} \mathrm{C} ;{ }^{1} \mathrm{H}$ NMR (DMSO- $d_{6}$ ) $\delta: 8.190$ (d, $J=9.0 \mathrm{~Hz}, 4 \mathrm{H}, \mathrm{ArH}), 7.493 \sim 7.532(\mathrm{~m}, 2 \mathrm{H}$, ArH), $7.355 \sim 7.393(\mathrm{~m}, 1 \mathrm{H}, \operatorname{ArH}), 7.275$ (d, $J=8.0 \mathrm{~Hz}$, 2H, ArH), 7.204 (d, $J=9.0 \mathrm{~Hz}, 4 \mathrm{H}, \operatorname{ArH})$; IR (KBr) $v$ : $3651(\mathrm{O}-\mathrm{H})$; 1513, $1348\left(\mathrm{NO}_{2}\right), 1599,1466,1396(\mathrm{C}=$ C), $1184(\mathrm{C}-\mathrm{N}) \mathrm{cm}^{-1}$. Anal. calcd for $\mathrm{C}_{18} \mathrm{H}_{13} \mathrm{~N}_{3} \mathrm{O}_{5}: \mathrm{C}$ 61.48, H 3.69, N 12.09; found C 61.50, H 3.70, N 12.06.

4-[二(对硝基苯基)氨基苯氧基]邻苯二甲腈(2)的合 成与表征: 将化合物 $\mathbf{1}(0.8 \mathrm{~g})$ 和 4-硝基邻苯二甲腈 $(0.4$ g)溶解在 $20 \mathrm{~mL}$ 二甲基亚砜中(DMSO), 边摚拌边加入 $\mathrm{LiOH} \cdot \mathrm{H}_{2} \mathrm{O}(0.8 \mathrm{~g})$, 在 $40{ }^{\circ} \mathrm{C}$ 下摚拌 $48 \mathrm{~h}$. 将反应混合物 倒入 $200 \mathrm{~mL}$ 冰水中析出沉淀, 抽滤, 滤饼用水洗涤, 再 用无水乙醇重结晶、干燥. 产量 $0.75 \mathrm{~g}$, 产率 $62.5 \%$. m.p. $>200{ }^{\circ} \mathrm{C} ;{ }^{1} \mathrm{H}$ NMR (DMSO- $\left.d_{6}\right) \delta: 9.0(\mathrm{~d}, J=8.0 \mathrm{~Hz}, 2 \mathrm{H}$, $\mathrm{ArH}$ ), $8.65 \sim 8.18(\mathrm{~m}, 5 \mathrm{H}, \mathrm{ArH}) 7.0 \sim 6.62(\mathrm{~m}, 8 \mathrm{H}, \mathrm{ArH})$; IR (KBr) v: $2238(\mathrm{C} \equiv \mathrm{N})$; 2361, 1240, $1082(\mathrm{C}-\mathrm{O}-\mathrm{C})$, 1599, 1466, $1396(\mathrm{C}=\mathrm{C})$. Anal. calcd for $\mathrm{C}_{26} \mathrm{H}_{15} \mathrm{~N}_{5} \mathrm{O}_{5}: \mathrm{C}$ 65.38, H 3.18, N 14.79; found C 65.40, H 3.20, N 14.80.

4-(8-氧基喹啉)邻苯二甲腈(3)的合成与表征: 将 4硝基邻苯二甲腈 $(0.866 \mathrm{~g}, 5 \mathrm{mmol})$ 和 8 -差欮基喹啉 $(0.80 \mathrm{~g}$, $5 \mathrm{mmol}$ )溶解在 $10 \mathrm{~mL} \mathrm{DMF}$ 中, 待完全溶解后, 加入无 水 $\mathrm{K}_{2} \mathrm{CO}_{3}(1.0 \mathrm{~g}, 7 \mathrm{mmol})$. 剧烈搅拌, $2 \mathrm{~h}$ 后补加 $0.5 \mathrm{~g}$ $\mathrm{K}_{2} \mathrm{CO}_{3}$. 反应在氮气保护下剧烈摚拌 $32 \mathrm{~h}$, 温度为 60 ${ }^{\circ} \mathrm{C}$. 反应结束后将反应的混合物倒入冰水中, 并搅拌 15 $\min$, 过滤沉淀物, 并用蒸馏水洗涤. 用无水甲醇重结 晶, 并干燥. 产量 $1.43 \mathrm{~g}$, 产率 $85.8 \%$. 浅灰色固体, m.p. $212{ }^{\circ} \mathrm{C} ;{ }^{1} \mathrm{H}$ NMR (DMSO- $\left.d_{6}\right) \delta: 7.19 \sim 8.35(\mathrm{~m}, 7 \mathrm{H}$, ArH); IR (KBr) v: 3101, 3074, 3038 (H-Ar), 2259 (C $\equiv$ $\mathrm{N}), 1587,(\mathrm{Ar}-\mathrm{C}=\mathrm{C}) ; 1254(\mathrm{Ar}-\mathrm{O}-\mathrm{Ar}) \mathrm{cm}^{-1}$. Anal. calcd for $\mathrm{C}_{17} \mathrm{H}_{9} \mathrm{~N}_{3} \mathrm{O}$ : C 75.26, H 3.35, N 15.48; found $\mathrm{C}$ 75.28, H 3.32, N 15.50.
3.3.2 2-(二(对硝基苯基)氨基苯氧基)-9,16,23-三(8喹啉氧)锌酞菁(TQPc)的合成与表征

将 2 与 3 按 $1: 3$ 的物质的量比混合(分别为 $0.30 \mathrm{~g}$, $0.51 \mathrm{~g}$ ), 加入乙酸锌(或其它金属的盐), 在研钵中充分 研细使每种化合物均匀混合，将混合物加入到三口烧瓶 中, 加入 $30 \mathrm{~mL}$ 左右的正戊醇, 边搅拌边加入 $6 \sim 10$ 滴 $\mathrm{DBU}$, 在氮气保护下油浴加热, 使温度缓慢升至 145 ${ }^{\circ} \mathrm{C}$, 继续摚拌 $8 \sim 12 \mathrm{~h}$, 然后冷却至室温, 冷却后缓慢加 入一定量的甲醇摚拌 $15 \mathrm{~min}$, 过滤沉淀，再用甲醇充分 洗涤, 得到墨绿色固体, 真空干燥. 进行柱层析分离提 纯, 用 $80 \sim 100$ 目硅胶, $V($ 氯仿 $): V($ 乙酸乙酯 $)=10: 1$ 洗下第一带为四喹啉氧基锌酞菁. 之后用 $V$ (氯仿) : $V($ 乙酸乙酯 $)=1: 1$ 洗下第二带, 真空干燥后再一次进 行柱层析分离提纯, 用 $80 \sim 100$ 目硅胶, 用 $V$ (氯仿)： $V($ 乙酸乙酯 $)=1: 2$ 洗脱, 收集两个色带, 真空干燥后 得到两种颜色相近的墨绿色固体, 经表征得出, 第二带 为目标产物. 产量 $0.19 \mathrm{~g}$, 产率 $23.46 \%$. 绿色固体, m.p. $>200{ }^{\circ} \mathrm{C} ;{ }^{1} \mathrm{H}$ NMR (DMSO- $\left.d_{6}\right) \delta: 8.85 \sim 8.62(\mathrm{~m}, 4 \mathrm{H}$, $\mathrm{ArH}), 8.58 \sim 8.46(\mathrm{~m}, 3 \mathrm{H}, \mathrm{ArH}), 7.9 \sim 7.45(\mathrm{~m}, 35 \mathrm{H}, \mathrm{ArH})$; IR $(\mathrm{KBr}) v: 1482,1666(\mathrm{C}=\mathrm{N}), 2361,1240,1082(\mathrm{C}-$ $\mathrm{O}-\mathrm{C}), 1599,1466,1396(\mathrm{C}=\mathrm{C}), 1110(\mathrm{C}-\mathrm{N}) \mathrm{cm}^{-1}$. Anal. calcd for $\mathrm{C}_{77} \mathrm{H}_{42} \mathrm{~N}_{14} \mathrm{O}_{8} \mathrm{Zn}$ : C 68.20, H 3.10, N 14.47; found C 67.93, H 3.27, N 14.43.

\subsection{3 溶液的配制}

准确称取一定量的 2-(二(对硝基苯基)氨基苯氧 基)-9,16,23-三(8-喹啉氧)锌酞菁(TQPc) 2 份, 分别溶于 DMF, $\mathrm{CH}_{2} \mathrm{Cl}_{2}$ 中, 确定各自的浓度, 然后再将每种溶液 稀释成不同的浓度, 分别测定它们的吸收光谱, 之后计 算 $\varepsilon_{\mathrm{e}}, \varepsilon_{e}^{n} \cdot c_{0}^{n-1}, \varepsilon, K$ 等.

\section{References}

[1] O'regan, B.; Grätzel, M. Nature 1991, 353, 737.

[2] Hagfeldt, A.; Grätzel, M. Chem. Rev. 1995, 95, 49.

[3] Dai, S., Y.; Wang, K., J.; Weng, J.; Sui, Y., F.; Huang, Y.; Xiao, S., F. Solar Energy Mater. Solar Cells 2005, 85, 447.

[4] Horiuchi, T.; Miura, H.; Uchida, S. J. Photochem. Photobiol. A: Chem. 2004, 164, 29

[5] Li, C., Y.; Yang, X., C.; Chen, R., K.; Pan, J., X; Tian, H., N.; Zhu, H., J.; Wang, X., N.; Hagfeldt, A.; Sun, L., C. Solar Energy Mater. Solar Cells 2007, 91, 1863.

[6] Qin, H.; Wenger, S.; Xu, M.; Gao, F.-F.; Jing, X.-Y.; Wang, P.; Zakeeruddin, S. M.; Grätzel, M. J. Am. Chem. Soc. 2008, 130, 9202.

[7] Dastoor, P. C.; McNeill, C. R.; Frohne, H.; Foster, C. J.; Dean, B.; Fell, C. J.; Belcher, W. J.; Campbell, W. M.; Officer, D. L.; Blake, L. M.; Thordarson, P.; Crossley, M. J.; Hush, N. S.; Reimers, J. R. J. Phys. Chem. C 2007, 111, 15415.

[8] Deng, H.-H.; Mao, H.-F.; Shen, Y.-C.; Lu, Z.-H.; Xu, H.-J. Acta Chim. Sinica 1999, 57, 1199 (in Chinese).

(邓慧华，毛海舫，沈耀春，陆祖宏，许慧君，化学学报，1999, 
57, 1199.)

[9] Hou, Y. J.; Xie, P. H.; Zhang, B. W.; Cao, Y.; Xiao, X. R.; Wang, W. B. Inorg. Chem. 1999, 38, 6320.

[10] Clifford, J. N.; Palomares, E.; Nazeeruddin, M. D. K.; Thampi, R.; Grätzel, M.; Durrant, J. R. J. Am. Chem. Soc. 2004, 126, 5670.

[11] Kong, F.-T.; Dai, S.-Y.; Wang, K.-J. Chemistry 2005, (5), 338 (in Chinese).

(孔凡太, 戴松元, 王孔嘉, 化学通报, 2005, (5), 338.)

[12] Wu, D.; Shen, Z.; Xue, Z.-L.; You, X., Z. Chin. J. Inorg. Chem. 2007, 23, 1 (in Chinese). (吴迪, 沈珍, 薛兆历, 游效曾, 无机化学学报, 2007, 23, 1.)

[13] Wang, Q.; Campbell, W. M.; Bonfantani, E. E.; Jolley, K. W.; Officer, D.; Walsh, P. J.; Gordon, K.; Baker, R. H.; Nazeeruddin, M. K.; Grätzel, M. J. Phys. Chem. B 2005, 109, 15397.

[14] Chen, W.; Duan, W.-B.; He, C.-Y.; Zuo, X.; Wu, Y.-Q. Chin. J. Inorg. Chem. 2005, 21, 1880 (in Chinese).

(陈伟, 段武彪, 贺春英, 左霞, 吴谊群, 无机化学学报, 2005 , 21, 1880.)

[15] Hammer, R. P.; Owens, C. V.; Hwang, S. H.; Sayes, C. M.; Soper, S. A. Bioconjugate Chem. 2002, 13, 1244.

[16] Campbell, W. M.; Jolley, K. W.; Wagner, P.; Wagner, K.; Walsh, P. J.; Gordon, K. C.; Mende, L. S.; Nazeeruddin, M. K.; Wang, Q.; Grätzel, M.; Officer, D. L. J. Phys. Chem. C 2007, 111, 11760.

[17] Bai, Q.-L.; Zhang, C.-H.; Cheng, C.-H.; Li, W.-C.; Du, G.-T. Acta Chim. Sinica 2011, 69, 949 (in Chinese).

(白青龙, 张春花, 程传辉, 李万程, 杜国同, 化学学报, 2011, 69, 949.)

[18] Yu, X.-W.; Zhan, C.-L.; Huang, Y. Chin. J. Org. Chem. 2012, 32, 770 (in Chinese).

(俞孝伟, 詹传郎, 黄彦, 有机化学, 2012, 32, 770.)

[19] Zhang, D.; Zhang, X.-J.; Zhang, L.; Mao, L.-J. Bull. Korean Chem.
Soc. 2012, 33, 1

[20] Pei, J. Ph.D. Dissertation, Nankai University, Tianjin, 2009 (in Chinese).

(裴娟, 博士论文, 南开大学, 天津, 2009.)

[21] Peng, Y.-R.; Huang, F.-H. Strait Pharm. J. 2003, 15, 53 (in Chinese).

(彭亦如，黄风华，海峡药学, 2003, 15, 53.)

[22] Liu, Q.-H.; Fu, Q.; Yang, J.; Ma, J.-C.; Li, W.-L.; Wang, X. J. Mol. Struct. 2010, 963, 41

[23] Yenilmez, H.-Y.; Özcesmeci, İ.; Okur, A. İ.; Gül, A. Polyhedron 2004, 23, 787.

[24] Liu, L.-W. M.S. Thesis, Zhejiang University, Hangzhou, 2008 (in Chinese). (刘立维, 硕士论文, 浙江大学, 杭州, 2008.)

[25] Li, W.-L. M.S. Thesis, Northeast Normal University, Changchun, 2010 (in Chinese). (李伟利, 硕士论文, 东北师范大学, 长春, 2010.)

[26] Sastre, A.; Torres, T.; Hanack, M. Tetrahedron Lett. 1995, 36, 8501.

[27] Zhang, X.-J.; Mao, L.-J.; Zhang, D.; Zhang, L. J. Mol. Struct. 2012, $1022,153$.

[28] Giribabu, L.; Vijay, K. C.; Gopal, R. V.; Yella, R. P. Solar Energ. Mater. Solar Cells 2007, 91, 1611.

[29] Hagfeldt, A.; Grätzel, M. Chem. Rev. 1995, 95, 49.

[30] Liu, L.-W.; Shi, M.-M.; Deng, D.; Wang, M.; Chen, H.-Z. Acta Chim. Sinica 2008, 66, 2163 (in Chinese). (刘立维，施敏敏，邓丹，汪茫，陈红征，化学学报，2008，66, 2163.)

[31] Zhang, T.-L.; Yan, J.-M. Acta Chim. Sinica 2000, 58, 981 (in Chinese).

(张天莉, 严继民, 化学学报, 2000, 58,981 .)

(Qin, X.) 